\title{
L-/T-type Ca channel blockers for kidney protection: ready for sophisticated use of $\mathrm{Ca}$ channel blockers
}

\author{
Koichi Hayashi \\ Hypertension Research (2011) 34, 910-912; doi:10.1038/hr.2011.74; published online 14 July 2011
}

$\mathrm{M}$ uch attention has been paid to the implication of chronic kidney disease (CKD) as a major risk factor for cardiovascular disease. In diabetic patients, microalbuminuria constitutes a critical prognostic factor for the development of nephropathy as well as a surrogate marker for cardiovascular events. It is well established that the blockade of the renin-angiotensin system with angiotensin-converting enzyme inhibitors or angiotensin receptor blockers (ARBs) inhibit the progression of diabetic nephropathy and hypertension, and is actually recommended as a first choice drug for the treatment of diabetes and CKD. Despite the use of maximal doses of these agents, it is not uncommon for intractable hypertension to persist, requiring additional medication. As indicated in the JSH $2009^{1}$ guideline, calcium channel blockers (CCBs) are a representative class of add-on drugs and are believed to be extremely reliable in reducing blood pressure to an optimal level.

Several clinical studies, including NICE Combi, ${ }^{2}$ have shown that adding $\mathrm{CCB}$ to ARB effectively reduces blood pressure and urinary protein excretion. The rationale for renal protective action of CCB is considered multifactorial. Although the reduction in systemic blood pressure constitutes a major component, the role of intraglomerular pressure is also a critical factor in determining the development of nephropathy. On the basis of renal microvascular action, the conventional CCBs, such as nifedipine and amlodipine, preferentially dilate the afferent arteriole, whereas the efferent arteriolar action is modest. $^{3}$ The beneficial action of CCBs

Dr K Hayashi is at the Department of Internal Medicine, School of Medicine, Keio University, 35 Shinanomachi, Shinjuku-ku, Tokyo 160-8582, Japan.

E-mail: khayashi@sc.itc.keio.ac.jp through the reduction in systemic blood pressure thus may be countered by afferent arterial dilation, whereby systemic blood pressure is directly transmitted to the glomerulus. Indeed, the MARVAL trial indicates that amlodipine increases microalbuminuria in type 2 diabetic nephropathy. ${ }^{4}$ Furthermore, amlodipine failed to reduce the incidence of renal end point in diabetic nephropathy (irbesartan diabetic nephropathy trial ${ }^{5}$ ), or was shown to be less effective in protecting against the progression to renal end points in patients with stage 4 CKD (CASE-J subanalysis ${ }^{6}$ ). These observations clearly indicate the importance of glomerular hypertension as a pathogenetic factor for CKD progression.

In this issue of the journal, Abe et al. ${ }^{7}$ demonstrated important issues regarding the application of CCBs to CKD patients. They showed differing action of amlodipine and azelnidipine on urinary albumin excretion in diabetic patients who were already being treated with ARB. As the additional reductions in blood pressure were the same in the amlodipine as in the azelnidipine groups, the divergent renal effects observed may be derived from differences in pharmacological properties of these CCBs. It has now been established that CCBs, particularly those that have been developed in Japan, confer an intriguing action on renal microvascular beds.

Unlike conventional CCBs (for example, nifedipine and amlodipine), efonidipine exerts vasodilator action on both afferent and efferent arterioles, which subsequently reduces proteinuria and retards the progression of $\mathrm{CKD}^{3}$ Similar findings have been observed for other CCBs, including azelnidipine $^{8}$ and cilnidipine. ${ }^{9}$ Several investigators have attempted to explore the mechanism of the renal microvascular action of CCBs, and now have clarified the role of Ca channel subtypes as distinct target sites for CCBs. The conventional CCB class predominantly blocks L-type Ca channels that distribute preferentially at the afferent, but not efferent, arteriole (Figure 1). By contrast, newly identified classes of CCBs (efonidipine, benidipine, azelnidipine and cilnidipine) exert their inhibitory action through T-type or N-type Ca channels in addition to L-type Ca channels. Because T-type $\mathrm{Ca}$ channels prevail at both afferent and efferent arterioles and $\mathrm{N}$-type Ca channels are present at the nerve terminal innervating both arterioles, the inhibition of these $\mathrm{Ca}$ channels is likely to dilate afferent and efferent arterioles, resulting in the reduction in glomerular capillary pressure. The clinical study conducted by Abe et al., ${ }^{7}$ therefore, may reflect the pharmacological aspects of the Ca channel blockade in renal microcirculation, showing the divergent action of these CCBs on renal protection.

There is still some debate regarding the renal effects of add-on treatment with CCBs. As mentioned above, the NICE Combi study showed a decrease in proteinuria from the addition of nifedipine, in parallel with a substantial reduction in blood pressure (from 153 to $140 \mathrm{~mm} \mathrm{Hg}$ ). ${ }^{2}$ Ogawa et al. ${ }^{8}$ also showed that the addition of either azelnidipine or nifedipine decreases albuminuria in type 2 diabetic patients. Of importance, the slope of the correlation between the changes in albumin/creatinine ratio (ACR) and those in blood pressure was much steeper in the azelnidipine group than in the nifedipine group (Figure 2). Furthermore, with the same degree of blood pressure reduction, the decrease in urinary ACR in the azelnidipine group was greater than that in the nifedipine group. In the study by Abe et al., ${ }^{7}$ azelnidipine resulted in a greater decrease in albumin excretion (from 387 to $260 \mathrm{mg}$ per $\mathrm{gCr}$ ) than amlodipine (from 380 


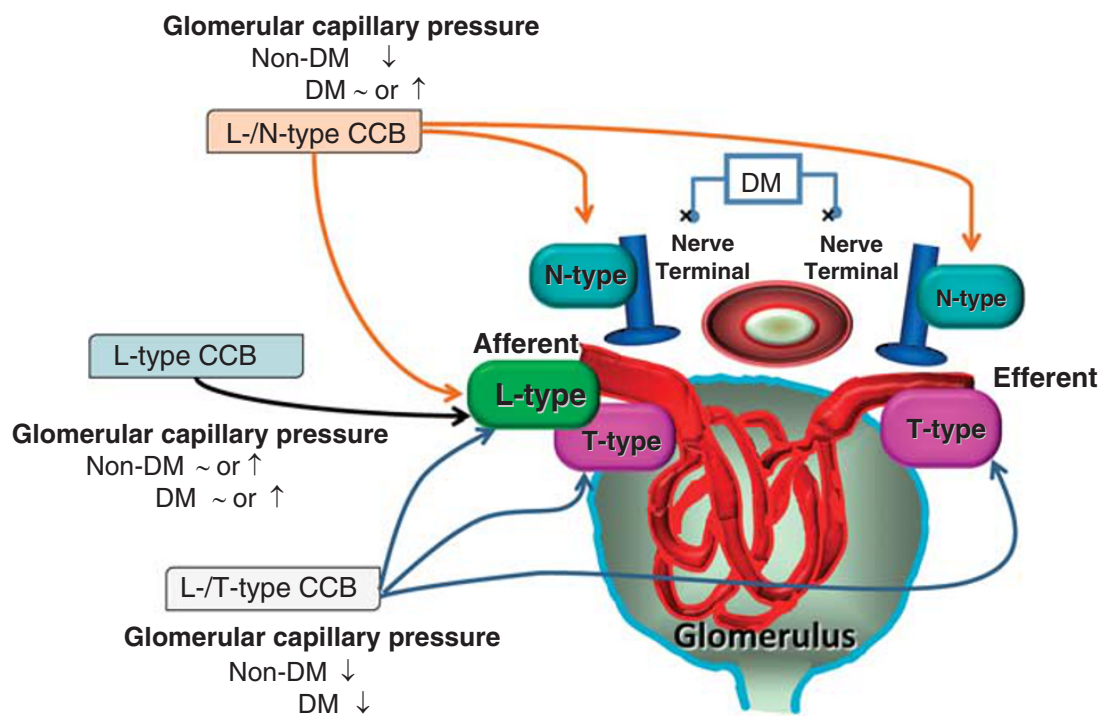

Figure 1 Distribution of Ca channel subtypes in the renal microcirculation. L-type Ca channel blockers (CCBs) predominantly cause dilation of the afferent arteriole. Subsequently, glomerular capillary pressure is elevated or unaltered, depending on the balance between afferent arteriolar resistance and systemic blood pressure. L-/T-type CCBs dilate both afferent and efferent arterioles, which leads to a blood pressure-independent decrease in glomerular capillary pressure. Similar renal microvascular and glomerular hemodynamic actions are observed with L-/N-type CCBs in non-diabetic (non-DM) nephropathy. In diabetic (DM) patients, however, the development of neuropathy blunts the $\mathrm{N}$-type Ca channel-mediated action of L-/N-type CCBs on renal arterioles, resulting in altered renal microvascular activity of this type of CCB.
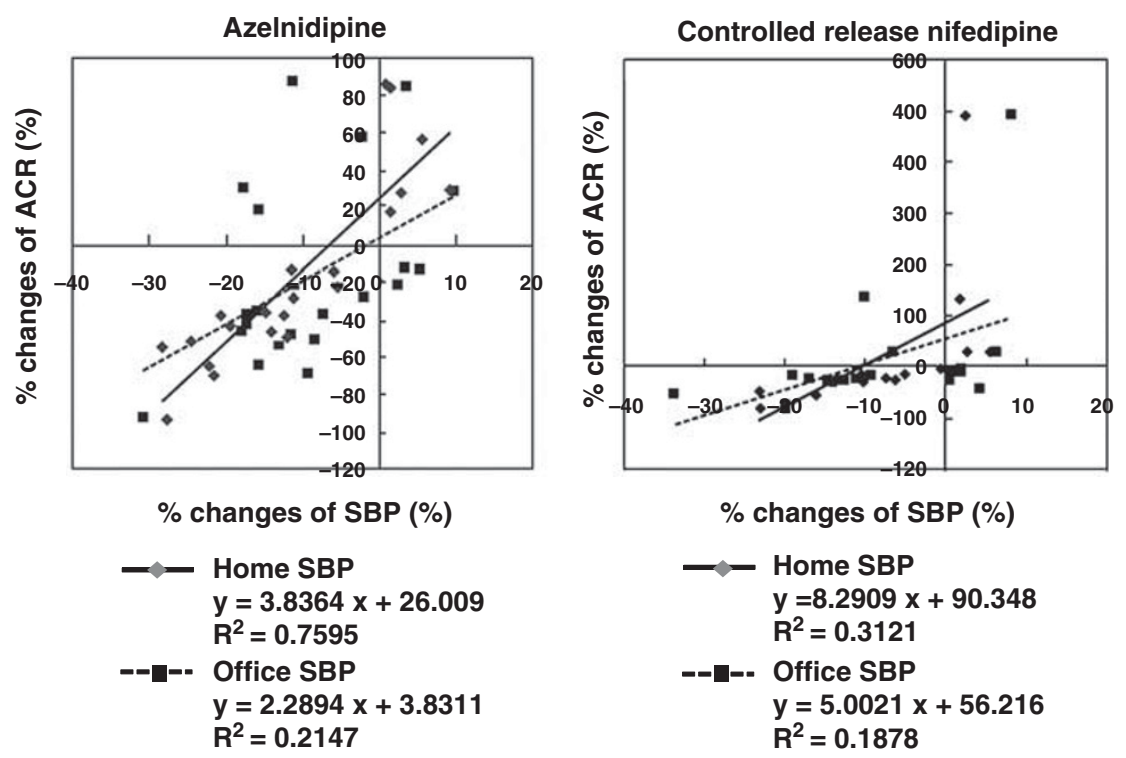

Figure 2 The correlation between the changes in systolic blood pressure and those in the albumin to creatinine ratio. Adapted from Ogawa et al. ${ }^{8}$ $\mathrm{ACR}$, urinary albumin to creatinine ratio; $\mathrm{CR}$, controlled release; SBP, systolic blood pressure.

to $302 \mathrm{mg}$ per $\mathrm{gCr}$ ) when blood pressure was reduced to a level of around $125 \mathrm{~mm} \mathrm{Hg}$. They also showed that the reduction of albuminuria due to benidipine was greater than that observed as a result of amlodipine in patients with CKD. ${ }^{10}$ In this regard, Omae et al. ${ }^{11}$ have demonstrated that treatment with L-/T-type CCBs (for example, benidipine, efonidipine and manidipine) is associated with better renal outcomes than L-type CCBs (for example, nifedipine, amlodipine and nicardipine) in non-diabetic CKD patients. Collectively, these observations indicate that L-/T-type CCBs are more potent than L-type CCBs in reducing proteinuria and the subsequent progression of CKD.

The role of other factors in the renal protective action of L-/T-type CCBs merits comment. The study by Abe et al. ${ }^{7}$ also evaluated the role of aldosterone and its association with urinary 18-hydroxy-2'deoxyguanosine or liver fatty acid-binding protein, which are markers for oxidative stress and proximal tubular injury, respectively. In this study, azelnidipine but not amlodipine reduced plasma aldosterone levels. They also reported analogous results showing a decrease in aldosterone levels by benidipine. ${ }^{10}$ Of note, aldosterone has recently been demonstrated to cause renal injury through multiple mechanisms, including tubulointerstitial fibrosis and inflammation, arteriolar sclerosis and glomerular hypertension. 
The blockade of aldosterone synthesis or release by L-/T-type CCBs ${ }^{12}$ may therefore confer beneficial action on CKD.

Interestingly, Abe et al. ${ }^{13}$ also demonstrated that an L-/N-type CCB, cilnidipine, reduced proteinuria as much as benidipine in CKD patients who were already being treated with $\mathrm{ARB}$, but exerted less antiproteinuric action than benidipine in patients with diabetes. In analogy, although cilnidipine was reported to be superior to amlodipine in reducing proteinuria in patients with $\mathrm{CKD}$, the changes in proteinuria by cilnidipine and those by amlodipine were not different in patients with diabetic nephropathy (CARTER $\left.\operatorname{trial}^{9}\right)$. Because the cilnidipine-induced efferent arteriolar action is mediated in large part by the blockade of $\mathrm{N}$-type $\mathrm{Ca}$ channels distributing at the nerve terminal, it is likely that the benefit conferred by the blockade of $\mathrm{N}$-type Ca channels is diminished in diabetic patients with neuropathy, when compared with T-type Ca channel blockade.

CCBs are widely used as antihypertensive agents, and are a reliable group of drugs because of their outstanding hypotensive action. As stated above, a growing body of evidence shows that CCBs with L-/T-type Ca channel blocking activity offer more beneficial action on CKD progression than CCBs with only L-type $\mathrm{Ca}$ channel action. Indeed, important roles for T-type Ca channel blockade have now been recognized in the field of nephrology, hypertension and cardiology. ${ }^{14}$ Unfortunately, CCBs with T-type Ca channel blocking activity are only clinically available primarily in Japan and South Korea and a large-scale clinical trial using this class of CCBs is lacking. This circumstance precludes worldwide recognition of this type of CCB. In this regard, a recent renal sub-analysis of the JATOS trial has demonstrated that 2-year treatment with efonidipine elevates estimated glomerular filtration rate (eGFR) in elderly hypertensive patients (65-85 year old) even when the patients have reduced renal function or diabetes at baseline. ${ }^{15}$ It is well known that renal function is likely to decrease in this subset of patients and diabetes further accelerates the progression of renal dysfunction. Although the study has no control subjects for the efonidipine-treated group and the results have been obtained based on a sub-analysis, the gradual elevation of eGFR throughout the trial period may reflect the renal protective action of this agent or could even suggest reversal of CKD.

In conclusion, dramatic advances have been made in understanding the Ca channel subtypes in the kidney. The discovery of CCBs that inhibit various $\mathrm{Ca}$ channel subtypes facilitates the clarification of the pharmacological implication of respective Ca channel subtypes, and may lead to the establishment of a therapeutic strategy for dealing with hypertension and CKD. Largescale clinical studies that compare the effects of L-type and L-/T-type CCBs would reinforce the recognition of the beneficial roles of the T-type Ca channel blockade in the treatment of hypertension with CKD. Although strict blood pressure control is indisputably required, it would be a matter for future investigation to establish which type of CCB is combined with renin-angiotensin system inhibitors to prevent the deterioration of renal function and halt the further progression of CKD.

1 Ogihara T, Kikuchi K, Matsuoka H, Fujita T, Higaki J, Horiuchi M, Imai Y, Imaizumi T, Ito S, Iwao H, Kario K, Kawano Y, Kim-Mitsuyama S, Kimura G, Matsubara H, Matsuura H, Naruse M, Saito I, Shimada K, Shimamoto K, Suzuki H, Takishita S, Tanahashi N, Tsuchihashi T, Uchiyama M, Ueda S, Ueshima H, Umemura S, Ishimitsu T, Rakugi $H$, on behalf of The Japanese Society of Hypertension Committee. The Japanese Society of Hypertension Guidelines for the Management of Hypertension (JSH 2009). Hypertens Res 2009; 32: 3-107.

2 Hasebe N, Kikuchi K, for the NICE Combi Study Group. Controlled-release nifedipine and candesartan low-dose combination therapy in patients with essential hypertension: the NICE Combi (Nifedipine and Candesartan Combination) Study. J Hypertens 2005; 23: 445-453.

3 Hayashi K, Wakino S, Sugano N, Ozawa Y, Homma K, Saruta T. $\mathrm{Ca}^{2+}$ channel subtypes and pharmacology in the kidney. Circ Res 2007; 100: 342-353.
4 Viberti G, Wheeldon NM, MicroAlbuminuria Reduction with VALsartan (MARVAL) Study Investigators. Microalbuminuria reduction with valsartan in patients with type 2 diabetes mellitus: a blood pressureindependent effect. Circulation 2002; 106: 672-678.

5 Lewis EJ, Hunsicker LG, Clarke WR, Berl T, Pohl MA, Lewis JB, Ritz E, Atkins RC, Raz I, Collaborative Study Group. Renoprotective effect of the angiotensinreceptor antagonist irbesartan in patients with nephropathy due to type 2 diabetes. N Engl J Med 2001; 345: 851-860.

6 Saruta T, Hayashi K, Ogihara T, Nakao K, Fukui T, Fukiyama K. Effects of candesartan and amlodipine on cardiovascular events in hypertensive patients with chronic kidney disease: subanalysis of the CASE-J study. Hypertens Res 2009; 32: 505-512.

7 Abe M, Maruyama N, Okada K, Matsumoto S, Matsumoto K, Soma M. Additive antioxidative effects of azelnidipine on angiotensin receptor blocker olmesartan treatment for type 2 diabetic patients with albuminuria. Hypertens Res 2011; 34: 935-941.

8 Ogawa S, Mori T, Nako K, Ito S. Combination therapy with renin-angiotensin system inhibitors and the calcium channel blocker azelnidipine decreases plasma inflammatory markers and urinary oxidative stress markers in patients with diabetic nephropathy. Hypertens Res 2008; 31: 1147-1155.

9 Fujita T, Ando K, Nishimura H, Ideura T, Yasuda G, Isshiki M, Takahashi K, on behalf of the Cilnidipine versus Amlodipine Randomized Trial for Evaluation in Renal Disease (CARTER) Study Investigators. Antiproteinuric effect of the calcium channel blocker cilnidipine added to renin-angiotensin inhibition in hypertensive patients with chronic renal disease. Kidney Int 2007; 72: 1543-1549.

10 Abe M, Okada K, Maruyama N, Matsumoto S, Maruyama T, Fujita T, Matsumoto K, Soma M. Benidipine reduces albuminuria and plasma aldosterone in mild-to-moderate stage chronic kidney disease with albuminuria. Hypertens Res 2011; 34: 268-273.

11 Omae K, Ogawa T, Nitta K. Influence of T-calcium channel blocker treatment on deterioration of renal function in chronic kidney disease. Heart Vessels 2009; 24: 301-307.

12 Isaka T, Ikeda K, Takada Y, Inada Y, Tojo K, Tajima N. Azelnidipine inhibits aldosterone synthesis and secretion in human adrenocortical cell line NCl-H295R. Eur J Pharmacol 2009; 605: 49-52.

13 Abe M, Okada K, Maruyama N, Matsumoto S, Maruyama T, Fujita T, Matsumoto K, Soma M. Comparison between the antiproteinuric effects of the calcium channel blockers benidipine and cilnidipine in combination with angiotensin receptor blockers in hypertensive patients with chronic kidney disease. Expert Opin Investig Drugs 2010; 19: 1027-1037.

14 Hart P, Bakris GL. Calcium antagonists: do they equally protect against kidney injury? Kidney Int 2008; 73: 795-796.

15 Hayashi K, Saruta T, Goto Y, Ishii M. Impact of renal function on cardiovascular events in elderly hypertensive patients treated with efonidipine. Hypertens Res 2010; 33: 1211-1220. 